\title{
TST-Negative Individuals Do Not Lack Anti-Mycobacterial Responsiveness
}

\author{
Aurélie Godel ${ }^{1,2, \S}$, Pascale Peyron ${ }^{1, \S}$, Marie-Pierre Puissegur ${ }^{1, \S}$, Catherine Botanch ${ }^{1}$, Bruno Payre ${ }^{3}$, \\ Sarah Khatibi ${ }^{2}$, Patrice Massip ${ }^{2}$, Bruno Marchou ${ }^{2}$ and Frédéric Altare ${ }^{*}, 1$
}

\author{
${ }^{I}$ IPBS-CNRS, 205 Route de Narbonne 31077 Toulouse Cedex 04 France (Drs Peyron, Puissegur, Botanch, Altare), \\ France \\ ${ }^{2}$ Service des Maladies Infectieuses de Toulouse, 1 place Baylac 31059 Toulouse cedex 04 (Drs. Godel, Khatibi, Prs. \\ Massip, Marchou), France \\ ${ }^{3}$ Faculté de médecine de Toulouse Rangueil; Centre de microscopie électronique appliquée à la Biologie; 133, route de \\ Narbonne 31062 Toulouse cedex 04 (Dr. Payre), France
}

\begin{abstract}
Tuberculin-skin-test (TST) is widely used for the diagnosis of Tuberculosis. Negative TSTs are occasionally found in individuals vaccinated or infected by Mycobacterium tuberculosis. We show that false negative results are due to a lack of the Tuberculin-specific immune response as replacing tuberculin by unselected M. tuberculosis antigens improved the efficiency of the assay.
\end{abstract}

Tuberculosis (TB) remains a major global public health concern, with one third of the world population infected, 9 million active cases and 2 million deaths every year [1]. Despite major progress in the development of new strategies for treating TB, the disease remains a great challenge for healthcare workers. Most people infected by M. Tuberculosis, the causative agent of $\mathrm{TB}$, remain asymptomatic (latent infection): the bacilli reside within granulomas, the histological hallmark of TB, and only $10 \%$ will develop a clinical disease during their life.

The tuberculin skin test (TST) is commonly used to help diagnosis of Mycobacterium Tuberculosis infection [2]. Purified protein derivative from M. Tuberculosis (PPD), also called Tuberculin, is injected intradermally, and the induction of a positive skin reaction is generally considered to indicate a latent or active TB, depending on the level of the skin reaction. In addition, positive tests are also found in patients after vaccination with Bacille Calmette-Guerin (BCG), or exposure to environmental mycobacteria. Negative test results may yet occur in a proportion of patients with active TB (10 to $25 \%$ ) [3,4]. These negative tests may find a biological explanation, as peripheral blood mononuclear cells (PBMC) from TB patients stimulated with Tuberculin (or PPD), release lower levels of IFN- $\gamma$ and IL-12 compared to PPD responsive (i.e. TST-positive) healthy subjects [5]. Qualitative TST responses may vary depending on the clinical presentation of TB, especially in children. Finally, it has been established that there is no relationship between tuberculin skin-reactivity, and protection of vaccinated individuals against the development of active Tubercu-

*Address correspondence to this author at the Department "Molecular Mechanisms of Mycobacterial Infections", Team "Molecular Physiology of Mycobacterial Granulomas", IPBS-CNRS UMR5089, 205, route de Narbonne, 31077 Toulouse cedex, France; Tel: (33) 5611754 63; Fax: (33) 5 611759 94; E-mail: frederic.altare@ipbs.fr

${ }^{\S}$ These authors contributed equally to the work. losis [6]. Thus, not only the TST does not distinguish between Tuberculosis infection and BCG vaccination, but hence it does not witness a mycobacterial infection (in false negative individuals), and finally does not appear as a correlate of protection either.

Recent advances have improved the specificity of the immune identification of latent TB, using a combination of antigens (early secreted antigenic target 6 (ESAT-6), and culture filtrate protein 10 (CFP-10) encoded by the RD1 locus of M. Tuberculosis which is absent from most nonpathogenic mycobacteria, including Bacille-Calmette-Guerin). The sensitivity has also been improved by the ELISPOT (T SPOT-TB assay), an ex vivo assay quantifying the $\gamma$-Interferon produced by T-cells stimulated with ESAT-6 and CFP- 10 . On another hand, the performance of the recently proposed Quantiferon tests (based on whole blood interferon $-\gamma$ release) has been shown to be negatively affected by patient's immune deficiency [7]. These serological and cytokine-based assays have yet to demonstrate an enhanced sensitivity and specificity when compared with the TST $[8,9]$. In BCGvaccinated individuals, false positive results occur with all these tests, and false negative tests due to anergy or immune deficiency are widely recognised [10-12]. Moreover, cost will be a critical factor in determining the global use of these new assays [13].

Pai and colleagues suggest combining the TST and $\gamma$ interferon assays to increase the sensitivity of the PPD-test and the specificity of RD1 antigens. This combination was used to confirm that the BCG vaccination gave significant protection against TB in children [14]. Yet, due to the numerous disadvantages of the TST previously discussed, we wondered whether we could find a better antigen than the Tuberculin to be used to give a more specific delayed-type hyper-sensitivity (DTH) reaction that could work in any individual. In this study, we thus investigated the possibility to replace Tuberculin by a more complex mixture of $M$. Tuberculosis (strain $\mathrm{H}_{37} \mathrm{Rv}$ ) antigens, obtained by mechanic 
disruption of the bacilli, which could possibly avoid false negative results. We used an in vitro model of human granulomas to compare the reactivity of human immunity to PPD and $M$. Tuberculosis antigens. This model reproduces in vitro a delayed-type hypersensitivity (DTH) reaction, as does the TST in vivo. This reaction is produced by the incubation of PBMC from healthy volunteers with sepharose beads coated with mycobacterial antigens, which produces a granuloma-like structure characteristic of the immune response to M. Tuberculosis [15-17].

Human blood samples were collected with informed consent from volunteers who had been BCG-vaccinated in infancy, and tested for their reactivity to PPD. For this purpose, PBMC from 100 volunteers (age 35-58, M/F: 61/39), including 85 PPD-positive individuals (Tubertest ${ }^{\circledR} 5 \mathrm{U}$,
$0.1 \mathrm{ml}$ ) and 15 PPD-negative individuals, were isolated, incubated with beads coated with PPD, or M. Tuberculosis extracts (prepared as described by Puissegur and colleagues [16]), glycin-coated beads serving as negative control for non specific reaction. The ability of the different individuals to develop a granulomatous reaction around the different beads was followed by optical microscopy (data not shown) and scanning electron microscopy (Fig. 1).

As expected, granulomas were found for PPD-responders (TST $>5 \mathrm{~mm}$ ), both around beads coated with PPD (Fig. 1A), and beads covered with M. Tuberculosis extracts (Fig. 1B). In the 15 PPD-nonresponders (TST $<5 \mathrm{~mm}$ ), no granulomas were ever found after incubation with PPD-coated beads (Fig. 1D), but important granulomatous reaction developed in response to M. Tuberculosis extracts (Fig. 1E).
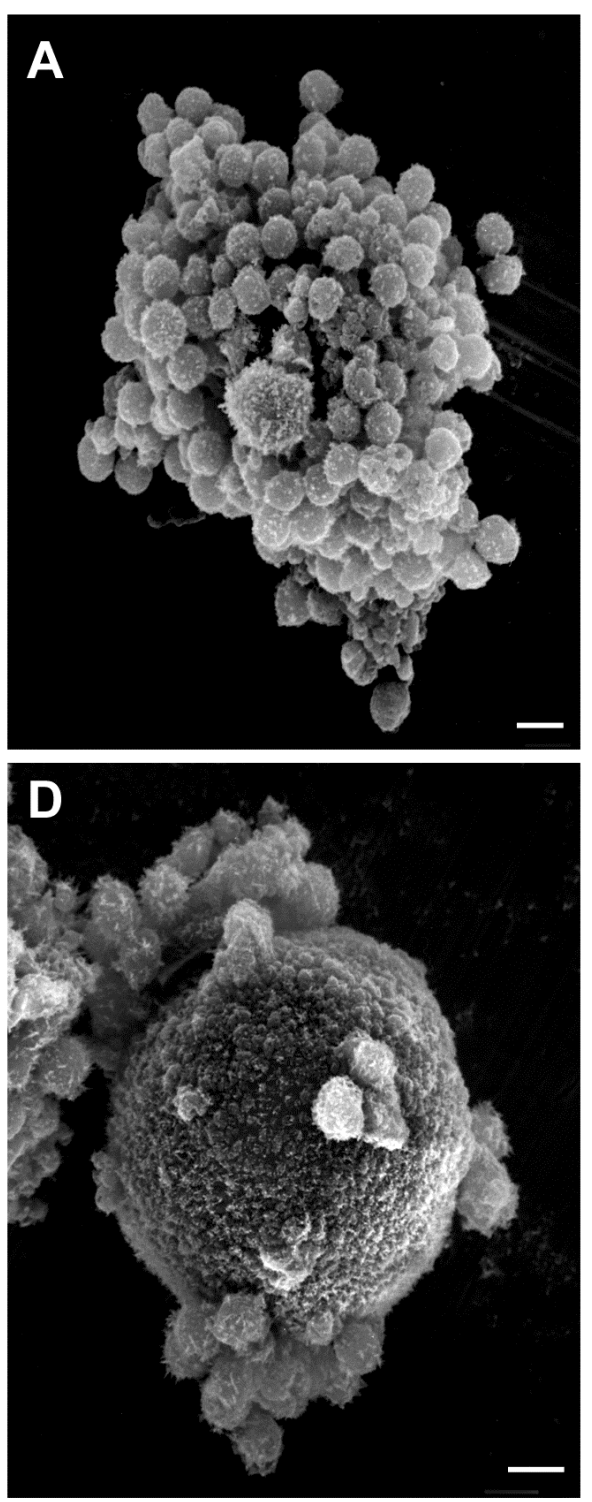
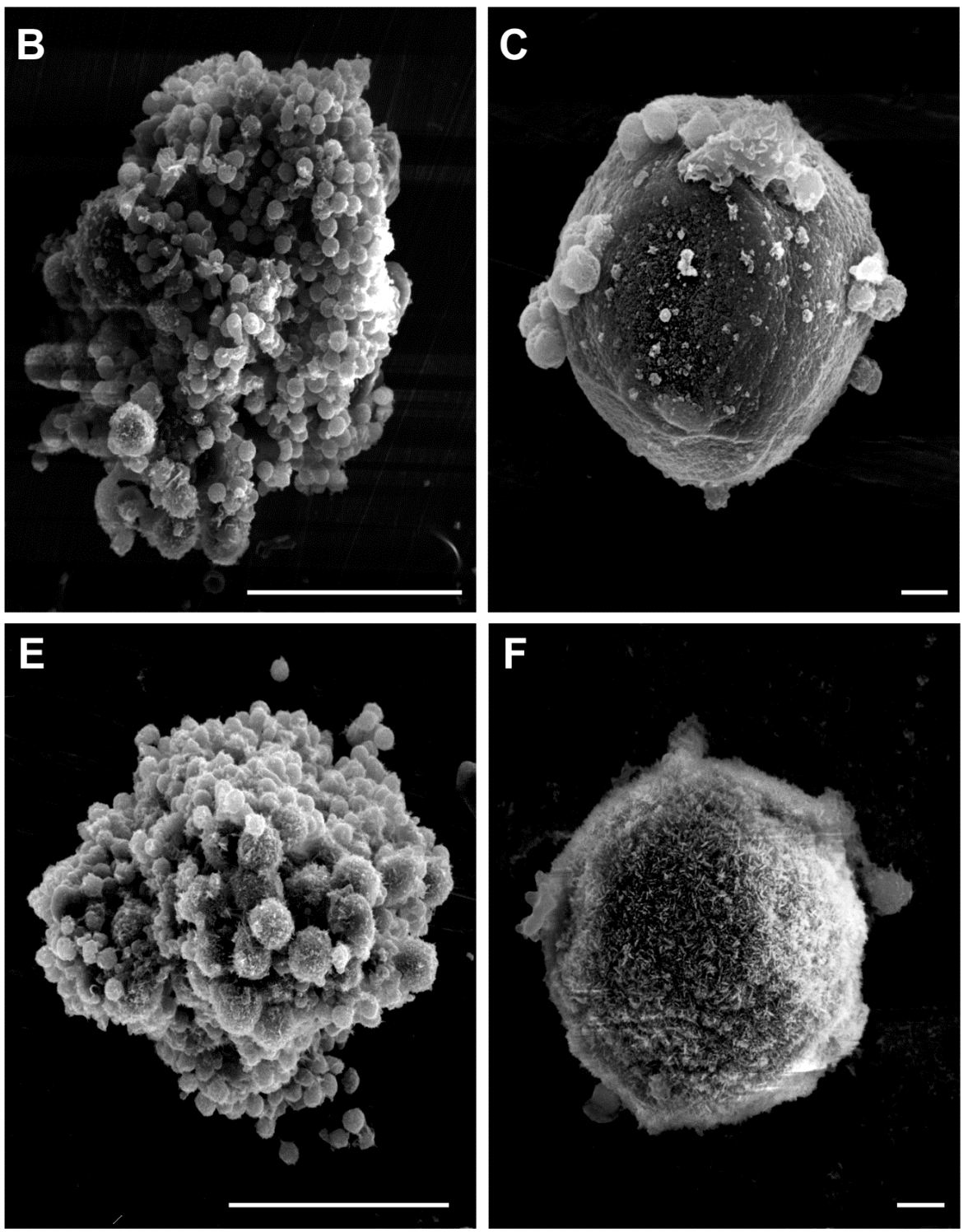

Fig. (1). Scanning electron micrographs of $\mathrm{H}_{37} \mathrm{Rv}$ surface extract-induced or PPD-induced granulomas. Beads covered with PPD $(\mathbf{A}, \mathbf{D})$ or surface extract from $\mathrm{H}_{37} \mathrm{Rv}(\mathbf{B}, \mathbf{E})$ were incubated with PBMC isolated from TST positive $(\mathbf{A}, \mathbf{B}, \mathbf{C})$ or negatives $(\mathbf{D}, \mathbf{E}, \mathbf{F})$ volunteers, all BCG vaccinated in infancy. Beads covered with Glycine $(\mathbf{C}, \mathbf{F})$ represent the negative control for non specific fixation. After 11 days of culture, granulomas were prepared and observed under a scanning electron microscope. Bar represent $5 \mu \mathrm{m}(\mathbf{A}, \mathbf{C}, \mathbf{D}, \mathbf{F})$ or $50 \mu \mathrm{m}(\mathbf{B}, \mathbf{E})$. All the analyses herein presented were performed according to the principles expressed in the Helsinki Declaration, with an informed consent of all volunteers. 
No cellular recruitment was observed with the negative control glycin-coated beads (Fig. 1C,F). A quantification of the granulomatous response has been possible by the definition of a granuloma index (Fig. 2A,B,C). The respective proportions of granulomas typical for each index has been determined for every TST- individual and presented in comparison to the index proportions of a representative TST + volunteer (Fig. 2D).

Noteworthy, the granulomatous response observed against M. Tuberculosis extracts is specific of the anti-mycobacterial response as no granulomatous response can be observed against $E$. Coli antigens, as previously described [16].

Nowadays, the TST remains the most widely used test worldwide to determine whether an individual has an immunological reactivity against mycobacterial antigens and for the diagnosis of TB. Pai and colleagues [14] suggested a combined use of PPD and a T-cell based ELISpot, using ESAT-6 and CFP-10 as antigens, for the diagnosis of latent TB. Here, we point out that the absence of response to PPD doesn't necessarily mean an energy to M. Tuberculosis anti- gens, but rather a selective defect in the response to PPD of given individuals. In this study, we have shown that PBMC from all subjects who have been vaccinated in childhood, are able to respond in vitro to $M$. Tuberculosis complex extracts by the induction of a DTH response, whatever their ability to react to PPD. We could yet not determine the specific antigens from M. Tuberculosis complex extracts, responsible for the induction of the DTH response. Our model of in vitro granulomas using beads coated with purified antigens from M. Tuberculosis such as ESAT-6 and CFP-10, could improve, in combination with the ELISPOT assay, the efficiency and reliability of the diagnosis of tuberculosis.

\section{ACKNOWLEDGEMENTS}

This work was supported by grants to PP from ANRS, and to FA from the INSERM (Programme Interface), the «Fondation pour la Recherche Médicale», "ACI Jeune Chercheur » from the " Ministère délégué à la Recherche et aux Nouvelles Technologies », the FP6 TB-VAC Project (LSHP-CT2003-503367).

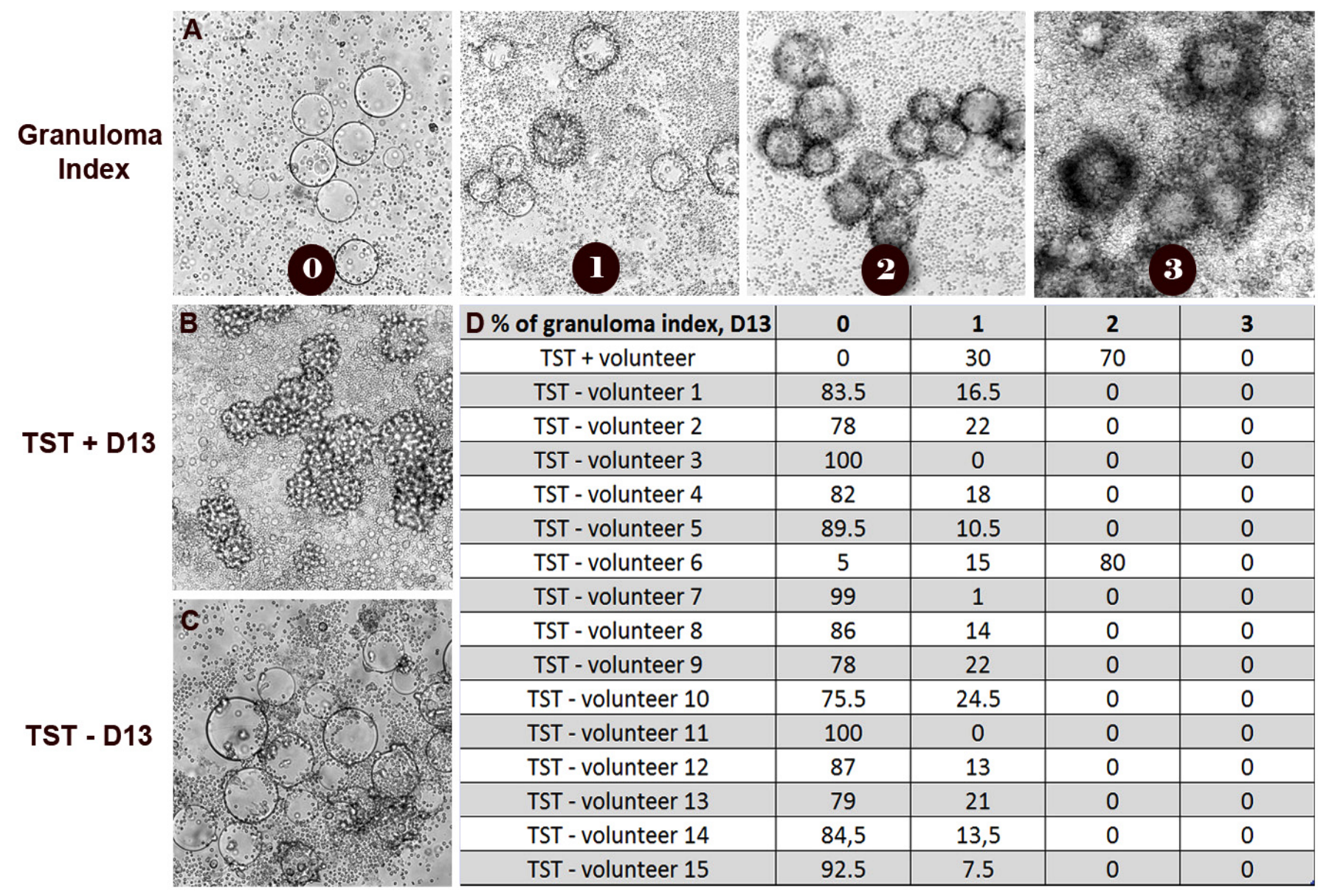

Fig. (2). Characterization of the granuloma index of TST negative individuals in response to PPD-beads. Beads covered with PPD were incubated with PPMCs from both TST negative (TST-) and TST positive (TST+) individuals. Pictures representing typical granuloma differentiation stages (index) are presented (A), magnification x200. Index 0: no cell recruitment around the beads. Index 1: several cells found on the beads up to a mono-layer cell recruitment with no cellular differentiation. Index 2: One to two cell layers around the beads, no sign of differentiation. Index 3: multi-layer cell recruitment, with strong macrophage differentiation, not visible on this picture. (B) Picture representative of granulomas found for TST+ individuals after 13 days of reaction (D13), magnification x200. (C) Picture representative of granulomas found for TST- individuals after 13 days of reaction (D13), magnification x200. (D) the proportions of the different granuloma index found after 13 days of reaction for the 15 TST- individuals and one TST+ representative individual, are presented. 


\section{REFERENCES}

[1] World Health Organization. Global Tuberculosis Control: surveillance, planning andfinancing. WHO Report. 2005 ISBN 924 156264: WHO/HTM/TB/2005.

[2] Huebner RE, Schein MF, Bass JB, Jr. The tuberculin skin test. Clin Infect Dis 1993; 17: 968-75.

[3] Fine PE, Sterne JA, Ponnighaus JM, Rees RJ. Delayed-type hypersensitivity, mycobacterial vaccines and protective immunity. Lancet 1994; 344: 1245-49.

[4] Wang L, Turner MO, Elwood RK, Schulzer M, FitzGerald JM. A meta-analysis of the effect of Bacille Calmette Guerin vaccination on tuberculin skin test measurements. Thorax 2002; 57: 804-9.

[5] Huygen K, Van Vooren JP, Turneer M, et al. Specific lymphoproliferation, gamma interferon production, and serum immunoglobulin $\mathrm{G}$ directed against a purified $32 \mathrm{kDa}$ mycobacterial protein antigen (P32) in patients with active tuberculosis. Scand J Immunol 1998; 27: 187-94.

[6] Menzies D. What does tuberculin reactivity after bacille CalmetteGuerin vaccination tell us? Clin Infect Dis 2000; 31 Suppl 3: S7174.

[7] Mazurek GH, Weis SE, Moonan PK, et al. Prospective comparison of the tuberculin skin test and 2 whole-blood interferon-gamma release assays in persons with suspected tuberculosis. Clin Infect Dis 2007; 45: 837-45

[8] Lalvani A, Richeldi L, Kunst H. Interferon gamma assays for tuberculosis. Lancet Infect Dis 2005; 5: 322-24; author reply 32527.

[9] Farhat M, Greenaway C, Pai M, Menzies D. False-positive tuberculin skin tests: what is the absolute effect of BCG and non- tuberculous mycobacteria? Int J Tuberc Lung Dis 2006; 10: 1192204.

[10] Pottumarthy S, Morris AJ, Harrison AC, Wells VC. Evaluation of the tuberculin gamma interferon assay: potential to replace the Mantoux skin test. J Clin Microbiol 1999; 37: 3229-32.

[11] Streeton JA, Desem N, Jones SL. Sensitivity and specificity of a gamma interferon blood test for tuberculosis infection. Int J Tuberc Lung Dis 1998; 2: 443-50.

[12] Vincenti D, Carrara S, Butera $\mathrm{O}$, et al. Response to region of difference 1 (RD1) epitopes in human immunodeficiency virus (HIV)-infected individuals enrolled with suspected active tuberculosis: a pilot study. Clin Exp Immunol 2007; 150: 91-98.

[13] Steingart KR, Ramsay A, Pai M. Commercial serological tests for the diagnosis of tuberculosis: do they work? Future Microbiol 2007; 2: 355-59.

[14] Soysal A, Millington KA, Bakir M, et al. Effect of BCG vaccination on risk of Mycobacterium tuberculosis infection in children with household tuberculosis contact: a prospective communitybased study. Lancet 2005; 366: 1443-51.

[15] Lay G, Poquet Y, Salek-Peyron P, et al. Langhans giant cells from M. tuberculosis-induced human granulomas cannot mediate mycobacterial uptake. J Pathol 2007; 211: 76-85.

[16] Puissegur MP, Botanch C, Duteyrat JL, et al. An in vitro dual model of mycobacterial granulomas to investigate the molecular interactions between mycobacteria and human host cells. Cell Microbiol 2004; 6: 423-33.

[17] Puissegur MP, Lay G, Gilleron M, et al. Mycobacterial lipomannan induces granuloma macrophage fusion via a TLR2-dependent, ADAM9- and beta1 integrin-mediated pathway. J Immunol 2007 ; 178: 3161-69.

(C) Godel et al.; Licensee Bentham Open.

This is an open access article distributed under the terms of the Creative Commons Attribution License (http://creativecommons.org/license/by/2.5/), which permits unrestrictive use, distribution, and reproduction in any medium, provided the original work is properly cited. 